\title{
MECHANICAL INDUCED BEAM MOTION IN SRRC STORAGE RING
}

\author{
D. J. Wang, C. K. Kuan, J. R. Chen \\ Synchrotron Radiation Research Center \\ No.1 R\&D Road VI. Science-Based Industrial Park, Hsinchu, Taiwan, R.O.C.
}

\begin{abstract}
It had been observed that the mechanical stability of some magnets had influences on the closed orbit in the storage ring. In this paper, a mechanical shaker in low vibration level was applied on the quadrupole magnet. The perturbation of the closed orbit was measured. The thermal induced distortion of magnet supporting structure was discussed. The motions of BPM and quadrupole magnet were measured. Finally some examples of mechanical induced beam motion were included.
\end{abstract}

\section{INTRODUCTION}

For the highest requirement of beamline users, the beam intensity fluctuation was pushed to a low limit. For the accelerator people, one possible reason of the intensity is electron beam orbit fluctuation. It includes the longterm drift $(<1 \mathrm{~Hz})$, in medium frequency $(<50 \mathrm{~Hz})$ vibration and higher frequency. Besides the ripple of magnet power supply, many mechanical related beam motions have been mentioned in other synchrotron facility. For example, the electron beam position monitor (BPM) moved by the vacuum chamber expansion [1], the thermal induced beam motion at ALS and SRRC [2-5], and the girder movement study at ESRF [6].

Global feedback system is usually used to correct the drift problem in low frequency. But by the limited number of BPM for feedback, the resolution and the mechanical displacement of BPM, for some beam ports especially not locked by BPM feedback locked system, small fluctuation may be possible. Therefore a better understanding of precision mechanical is essential for the stability of the beam.

In this paper, a systematic study of mechanical motion in micron meter level of key components in the storage was performed. A quadrupole magnet with high strength $2.8 \mathrm{~m}^{-2}$ was mechanical perturbed, the response of beam motion was recorded. Some examples of mechanical induced beam motion were included.

\section{THE POSSIBLE MECHANICAL FACTOR RELATED TO BEAM MOTION}

From the beam dynamics physics [7] a single point kick of strength, $\psi$, at $\mathrm{s}=\mathrm{o}$ along the circumference of a storage ring gives rise to a closed orbit displacement at position $\mathrm{s}$,

$$
\mathrm{y}_{\mathrm{co}}=\psi[\beta(0) \beta(\mathrm{s})]^{1 / 2} \cos [\phi(\mathrm{s})-\pi v] / 2 \sin \pi \nu
$$

where $\beta(0)$ and $\beta(\mathrm{s})$ are the betatron function at the location of the kick and the observation point respectively, $\phi(s)$ is the phase advance from the kick to the observation point and $v$ is the betatron tune. Kicks can arise from quadrupole magnet displacement and dipole field change as list below.

For quadrupole $\psi=\mathrm{K} \mathrm{L} \Delta, \Delta$ can be $\mathrm{x}$ or y displacement

For Dipole $\psi=\phi \theta$, if $\theta$ is the roll angle induce y motion

$\psi=\phi \Delta \mathrm{B} / \mathrm{B}, \mathrm{x}$ motion induced

$\mathrm{K}, \mathrm{L}$ and $\phi$ is the quadrupole strength, length and dipole angle.

$1 \mu \mathrm{m}$ displacement of one $\mathrm{Q} 2$ magnet $\left(\mathrm{K}=2.8 \mathrm{~m}^{-2}\right)$ will induce $6 \mu \mathrm{m}$ maximum along the circumference of the $\mathrm{BPM}$ of our ring. If all the Q2 are moved in phase the magnification factor is about 16[8]. The simulation will be given in the following figure. After a preliminary test of the dipole, thermal induced beam motion is much less than the quadrupole motion. For the beamline user, the vertical orbit motion is more critical than in horizontal direction when they do the high-resolution experiment. From the viewpoint of mechanical, the possible mechanical displacement can be breakdown as following.

A. Magnet girder: All the quadrupole magnets and BPM are mounted to the steel girder more than 2 meters long. It is fixed at two ends by two steel pedestal grouted to floor. A thermal induced distortion of girder in 2-3 $\mu \mathrm{m}$ will be induced when the air temperature variation in $0.5^{\circ} \mathrm{C}$ by the constraint of girder at both ends.

B. Quadrupole magnet: It is well known that the cooling water temperature of magnet will induce the beam motion [3]. The detail mechanism seems not clear.

\section{MEASUREMENT AND TEST METHOD}

1. To monitor the mechanical motion of key components in the ring, more than 50 position sensors were installed in the superperiod 2 of the storage ring. Quadrupole magnets, BPM were measured with potentiometer about $0.2 \mu \mathrm{m}$ resolution in 3 directions. The stainless steel support was about $30 \mathrm{~cm}$ long and with good thermal insulation. The access measuring points of magnet and BPM were the outer housing. It was difficult to measure the real pole surface and inside. The girder was measured by the linear variable differential transformer (LVDT) in $0.1 \mu \mathrm{m}$ resolution. Its support was a low thermal 
expansion quartz rod about $80 \mathrm{~cm}$. One girder with 6 LVDT to measure the height change relative to ground. The angle change and distortion of the girder can be deduced from the raw data. The layouts of the LVDT are at the upper, middle and downstream in the front and back side. All the sensor readings were linked to archived system.

2. A piezoelectric shaker was installed under a quadrupole magnet Q2 (with strength $2.8 \mathrm{~m}^{-2}$ ). Vibration amplitude in $\mu \mathrm{m}$ level and different frequency were applied. The reading of BPM were collected and compared.

\section{RESULT AND DISCUSSION}

\subsection{The closed orbit perturbation by $R 1 Q 7$}

In the Fig. 1 a vertical movement of R1Q7 magnet by a piezoelectric actuator with step function each step about $0.5 \mu \mathrm{m}$ total range is about $4 \mu \mathrm{m}$ with global feedback turn off. A theoretical simulation result is also attached for comparison. The measurement data seems reasonable. In the same condition as above but with the feedback turn on the result is shown in the table 1 . It can be easily seen that the R1 BPM variations are bigger than other superperiod. It may be related to the mechanical perturbation in the R1. It seems the feedback system can isolate the perturbation in the R1. In general the variation of BPMs for feedback interlock were less than other BPMs. Therefore in the user time if the perturbation by the quadrupole magnet some beam ports will sense the fluctuation even the feedback system turn on.

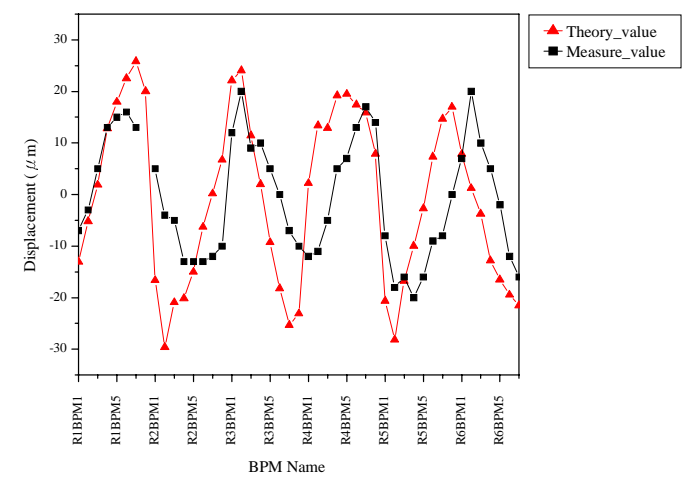

Figure 1: the electron beam orbit.

\subsection{Girder distortion and beam motion}

The girder deformation by the thermal fluctuation is shown in fig.2. By the air temperature variation about $0.3^{\circ} \mathrm{C}$ the height variation of girder in the upper, middle and downstream are $0.5 \mu \mathrm{m}, 1 \mu \mathrm{m}$ and $0.2 \mu \mathrm{m}$. The middle is large than both ends. It is suggested from the constrained by both ends and induced bow sag when the longitudinal expansion occurs. The periodicity of the middle height is coinciding with the ambient temperature. At the same time the BPM reading of this superperiod were shown in Fig. 3. It can be easily seen that the BPM $1,5,6,7$ locked by the feedback system without perturbation by the thermal variation. The others unlocked BPM 2,3,4,8 coincide the periodicity of thermal variation in the $2-3 \mu \mathrm{m}$. The difference between the girder and BPM movement may be from the magnification of quadrupole magnet. If the beam port behind the unlocked BPM then beam motion will occur. It seems one kind of long-term drift problem.

Table 1: The variation of BPMs reading with R1Q7 perturbation and feedback on (in $\mu \mathrm{m}$ )

\begin{tabular}{|l|l|l|l|l|l|l|}
\hline & R1 & R2 & R3 & R4 & R5 & R6 \\
\hline BPM1Y & $3^{*}$ & $1^{*}$ & 2 & $1^{*}$ & $1^{*}$ & 1 \\
\hline BPM2Y & 8 & 1.5 & $1^{*}$ & 1 & 2 & $2.5^{*}$ \\
\hline BPM3Y & 2 & 2.5 & 2 & 1 & $1^{*}$ & 7 \\
\hline BPM4Y & 3 & 2 & $1^{*}$ & 1 & 2 & $3^{*}$ \\
\hline BPM5Y & $3^{*}$ & $<1^{*}$ & $1^{*}$ & $<1^{*}$ & $2^{*}$ & $2^{*}$ \\
\hline BPM6Y & 3 & $1^{*}$ & 2 & $1.5^{*}$ & 2 & \\
\hline BPM7Y & 3 & $1^{*}$ & 1.5 & 1 & $3^{*}$ & 2 \\
\hline BPM8Y & & 2 & $1^{*}$ & $1.5^{*}$ & 2 & \\
\hline BPM9Y & $1^{*}$ & 1 & & 1.5 & & \\
\hline
\end{tabular}

*stand for the BPM used by global feedback

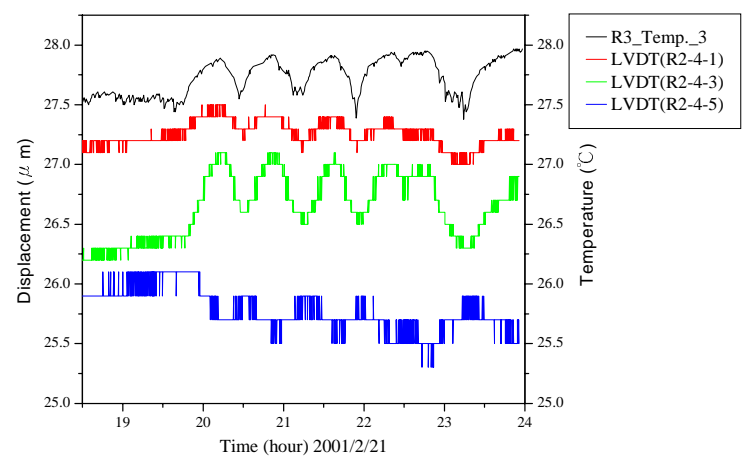

Figure 2: Girder deformation by the thermal fluctuation.

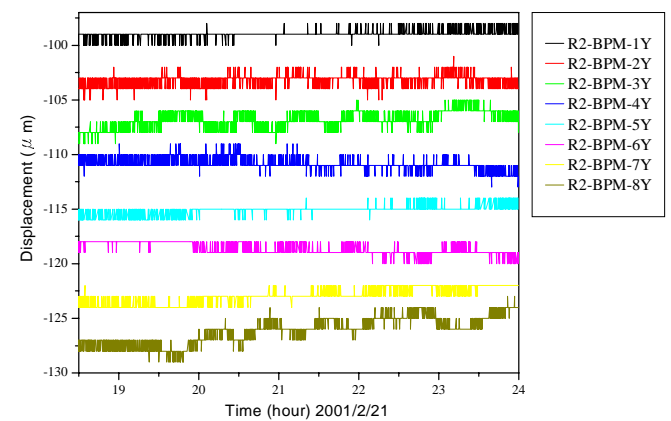

Figure 3: BPM reading as the same time of Fig. 2.

Another case of girder distortion is as following. During the Monday shutdown for 3 hours the entire thermal load is released. The air temperature is changed about $0.3^{\circ} \mathrm{C}$. The girder distortion is shown in Fig. 4. The height of girder change reached more than $5 \mu \mathrm{m}$ and lasted for more than 10 hours to reach equilibrium. Comparing Fig. 2 and Fig. 4 with the same thermal fluctuation the height deformation behaviors are different. It is related to the actual temperature or the time constant of the girder.

From the mechanical design the thermal induced distortion can be treated by 2 approach one is to release the constraint by kinematic design another is to increase the thermal time constant by thermal insulation. Good results have got in the preliminary test in the laboratory. 


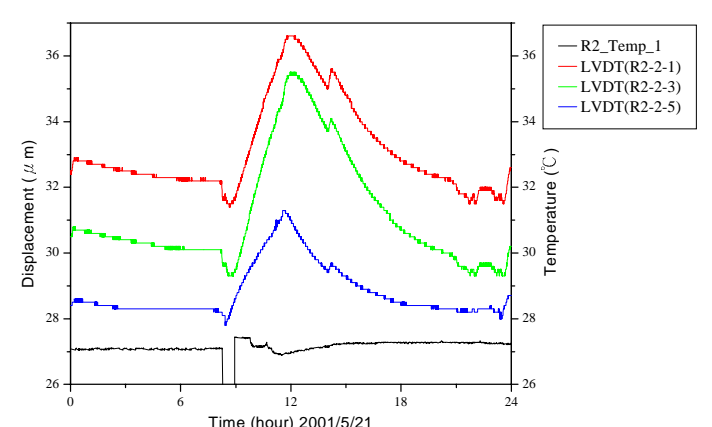

Figure 4: Girder distortion measured by LVDT sensors.

\subsection{The motion of BPM relative to girder}

In Fig. 5 the BPM mechanical position in 3 directions were recorded more than 20 hours. It is clear to find that the BPM position relative to girder was stable in $1 \mu \mathrm{m}$ in one shift. Vertical direction was disturbed by the injection and degaussing during the shift change. The driving force of the vertical change may be from the chamber expansion. In general the stiffness of the BPM support is good enough.



Figure 5: the BPM mechanical position readings.

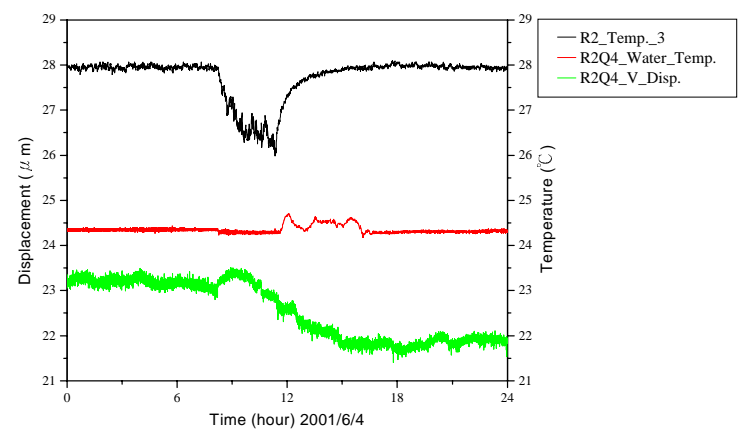

Figure 6: the vertical motion of the quadrupole magnet.

\subsection{The quadrupole motion}

A preliminary data of a quadrupole magnet vertical motion, air temperature and cooling water temperature are shown in Fig 6. In the Monday shutdown for 3 hours the air temperature changing $2^{\circ} \mathrm{C}$ the magnet vertical motion induce about $2 \mu \mathrm{m}$ motion. The water temperature variation $0.4{ }^{\circ} \mathrm{C}$ seems to have some correlation with the local variation of magnet motion. In the previous study [5], the water-cooling temperature has strong correlation with the beam motion. But the detail mechanism is not clear. It may be from thermal induced mechanical distortion. Because all the magnets were susceptible to the water temperature change, the magnification factor of the quadrupole were as high as 30 . It means the precision of measurement should be as low as $0.1 \mu \mathrm{m}$. It needs further study in the future.

\section{SUMMARY}

A $\mu \mathrm{m}$ level of mechanical perturbation on a quadrupole magnet was performed to compare the beam motion with and without feedback. Some BPM not interlocked will be perturbed even the feedback turning on. So the beam motion will occur. The major thermal induced mechanical distortion is on the girder. It influences the positioning of BPM and quadrupole (in $\mu \mathrm{m}$ level). A kinematic design and thermal insulation was applied to suppress the distortion.

The motion of quadrupole magnet was measured it was usually less than $0.5 \mu \mathrm{m}$ in one shift. A strong correlation between the magnet temperature and beam motion was observed. Is it also induced via mechanical distortion? More precise measurement and study will be performed.

\section{ACKNOWLEDGEMENT}

The authors would like to thank C.C.Kuo, H.P. Chang and P.J.Chou to discuss the beam dynamics physics and T.C.Tseng, C.H. Ho, C.J. Lin and S.Y. Perng to assist the measurement and Z.D. Tsai to install the Archive viewer.

\section{REFERENCES}

[1] Erik D. Johnson, Anne-Marie Fauchet, and Xiaohao Zhang, "Correlation of photon beam motion with vacuum chamber cooling on the NSLS x-ray ring", Rev. Sci. Instru. 63 (1), January 1992

[2] R.Keller, and H. Nishimura, "Orbit Stability of the ALS Storage Ring", Proceeding of PAC, 1997, Vancouver

[3] K.T. Hsu, et al, "Beam Orbit Stability at Taiwan Light Source", Proceeding of PAC, 1999, New York, USA

[4] H.M. Cheng, C.R. Chen, Z.D. Tsai, J.R. Chen "Utility Optimization for the Beam Orbit Stability at SRRC" Proceeding of PAC 1999, New York, USA

[5] J.R. Chen, Z.D. Tsai, C.K. Kuan, S.H. Chang, D. Lee, F.Y. Lin and D.J. Wang "Utility and Mechanical Component Stability at SRRC" Proceedings of $1^{\text {st }}$ international workshop on Mechanical Engineering Design of Synchrotron Radiation Equipment and Instrumentation" 2001,July 13/14 Switzerland PSI

[6] D. Martin, L. Farvacque, "Mechanical Induced Influences On the ESRF SR Beam" IWAA 1997, Chicago, USA

[7] S.Y.Lee "Introduction to Accelerator Physics"

[8] SRRC Design Handbook, 1992,Nov 